Chitnis, P.V., McLaughlan, J., Mamou, J., Murray, T.W., and Roy, R.A., “A photoacoustic sensor for monitoring temperature during HIFU exposures," 2009 International Symposium of Therapeutic Ultrasound Proceedings, AIP Conf. Proc. 1215, pp. 267-272; doi:http://dx.doi.org/10.1063/1.3367157 (2009).

\title{
A photoacoustic sensor for monitoring in situ temperature during HIFU exposures
}

\author{
Parag V. Chitnis*, James McLaughlan ${ }^{\dagger}$, Jonathan Mamou*, Todd Murray ${ }^{\dagger}$ and \\ Ronald A. Roy ${ }^{\dagger}$
}

${ }^{*}$ F. L. Lizzi Center for Biomedical Engineering, Riverside Research Institute, New York, NY 10038

${ }^{\dagger}$ Department of Mechanical Engineering, Boston University, Boston, MA 02215

\begin{abstract}
High-intensity focused ultrasound (HIFU) is a viable treatment option for small tumors. This study investigates the feasibility of employing a photoacoustic (PA) sensor to monitor the in situ temperature rise during HIFU exposures. The present method also provides means to simultaneously monitor inertial cavitation using the same sensor. Polyacrylamide phantoms with a cylindrical inclusion $(3 \times 20 \mathrm{~mm})$ of graphite $(0.01 \mathrm{~g} / \mathrm{ml})$ were heated using $30 \mathrm{~s}$ exposures from a $2 \mathrm{MHz}$ HIFU transducer. The transducer focus was aligned to the tip of a wire thermocouple embedded in the inclusion. A 532 $\mathrm{nm}$ pulsed laser was used to illuminate the inclusion. A $15 \mathrm{MHz}$ broadband transducer was employed as a passive receiver (PR) to detect the PA response, which was an ultrasonic pulse emanating from the inclusion due to thermoelastic expansion induced by optical absorption. The native temperature and PR signals were recorded before, during, and after HIFU exposure. Singular-value decomposition (SVD) was performed on the matrix consisting of the PR signals to extract temperature data. SVD-deduced, PA-based temperatures correlated well with the thermocouple measurements (RMS error $<5^{\circ} \mathrm{C}$ ). A temperature rise of $20^{\circ} \mathrm{C}$ corresponded to a $30 \%$ increase in PA amplitude. The PA temperature-measurement technique was able to track heating and cooling phases over a range of temperatures characteristic of HIFU-induced hyperthermia.
\end{abstract}

Keywords: Hyperthermia, HIFU, Photoacoustics, Thermometry>

PACS: 43.35.Ud

\section{INTRODUCTION}

Local hyperthermia is a viable treatment option for small tumors. High-intensity focused ultrasound (HIFU) is a hyperthermia modality that can provide noninvasive and non-ionizing treatment of tumors [1]. In HIFU therapy, absorption of ultrasonic energy at the focus leads to local heating, which results in thermal necrosis when the local temperature rises above $56^{\circ} \mathrm{C}$ for $2 \mathrm{~s}$ or more. Necrosis occurs at the focus without damaging surrounding healthy tissue [2]. Accurate, noninvasive, and real-time monitoring of in situ temperature is essential to assess the performance of and provide guidance for this treatment [3]. Mainstream research efforts to monitor local temperature and guide HIFU therapy concentrate on image-guided solutions based either on magnetic-resonance imaging [4], which is the current gold standard but cost prohibitive, or on ultrasound imaging $[5,6]$, which is economical but lacks precision and accuracy.

The present study investigates the feasibility of employing the photoacoustic (PA) effect for noninvasive thermometry during HIFU. PA imaging combines the spectral sensitivity of optical imaging with the spatial resolution of ultrasound; therefore, it potentially offers the ability to monitor temperature in real-time with high accuracy and precision $\left(0.5^{\circ} \mathrm{C}\right.$ or less) [7].

PA imaging relies on the local absorption of a brief $(\sim \mathrm{ns})$ monochromatic light pulse in the medium; optical absorption heats the medium and causes rapid thermoelastic expansion resulting in the generation of broadband ultrasonic waves, which can be detected and used to produce a PA image [8]. The PA pressure, $P$, at the absorption site is estimated by the following expressions:

$$
P=\left(\frac{\beta c^{2}}{C_{p}}\right) \mu_{a} F=G \mu_{a} F
$$

where $c$ is the sound speed, $\beta$ is the thermal-expansion coefficient, $C_{p}$ is the specific heat, $\mu_{a}$ is the absorption coefficient, and $F$ is the incident laser fluence. The expression $\left(G=\beta c^{2} / C_{p}\right)$ in Eq. 1 represents the efficiency of PA excitation, also known as the Grüneisen parameter. The constituents of $G$ are temperature dependent; consequently, the amplitude of the PA signal $(P)$ also is temperature dependent. PA amplitude has been shown to increase linearly with 


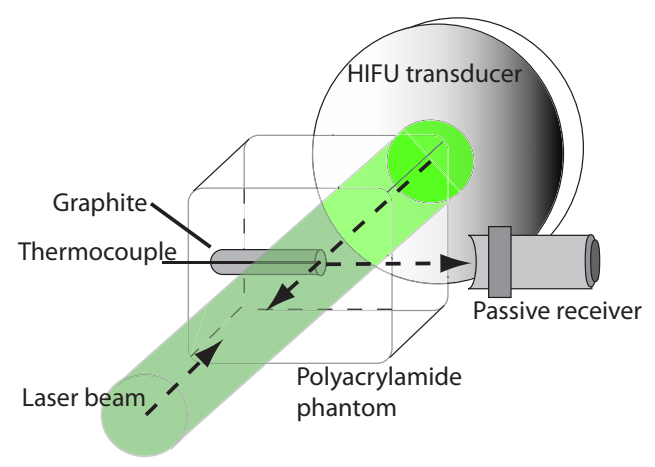

FIGURE 1. Schematic diagram of the experimental setup. The HIFU transducer and the PR transducer were confocal and orthogonal. The mutual focus was aligned at the tip of the thermocouple embedded in the graphite inclusion. An incident laser illuminated the volume around the thermocouple tip

temperature for water and excised tissue [7]. This study used PA-based thermometry to monitor in situ temperatures during HIFU exposures in tissue-mimicking phantoms.

\section{MATERIALS AND METHODS}

A $532 \mathrm{~nm} \mathrm{Nd}$ :Yag laser (Continuum Minilite-10, pulse duration $10 \mathrm{~ns}$, spot diameter $1.8 \mathrm{~mm}, 10 \mathrm{~Hz}$ repetition rate, fluence range of $0-17 \mathrm{~mJ} / \mathrm{cm}^{2}$ ) was used to generate PA emissions in Polyacrylamide gel phantoms. A $15 \mathrm{MHz}$ broadband transducer (V313, Olympus, focal distance $19 \mathrm{~mm}$, aperture $9 \mathrm{~mm}$ ) was employed as a passive receiver (PR) to detect these emissions. The PR transducer also served as a passive cavitation detector, thus, providing a congruent platform to simultaneously monitor temperature and cavitation in real-time. Details pertaining to the experimental setup and post-processing employed to produce temperature and cavitation results are presented below. A schematic diagram of the setup is shown in Fig 1.

\section{Experimental setup}

Polyacrylamide phantoms contained a cylindrical inclusion $(3 \times 20 \mathrm{~mm})$ of a concentration of graphite $(0.01 \mathrm{~g} / \mathrm{ml})$, which was heated using $30 \mathrm{~s}$ exposures from a $2 \mathrm{MHz}$ HIFU transducer (H-201, Sonic Concepts, focal distance $63 \mathrm{~mm}$, aperture $70 \mathrm{~mm}$ ). The transducer focus was aligned to the tip of a wire thermocouple (E-Type, diameter $0.13 \mathrm{~mm}$ ) embedded in the inclusion. HIFU intensities of $38 \mathrm{~W} / \mathrm{cm}^{2}, 89 \mathrm{~W} / \mathrm{cm}^{2}, 169 \mathrm{~W} / \mathrm{cm}^{2}$, and $543 \mathrm{~W} / \mathrm{cm}^{2}$ were employed with 5 min between each run. The temperature was recorded at a rate of $4 \mathrm{~Hz}$, using a data acquisition module (National Instruments, 9211A) connected to a PC. The PR signals were amplified using a $40 \mathrm{~dB}$ broadband preamplifier (Femto, HAS-X-2-40, $16 \mathrm{kHz}-2 \mathrm{GHz}$ ) and digitized (50 Ms/s) using an oscilloscope (LeCroy, WaverRunner LT342L). The oscilloscope was operated in sequence mode and triggered by a photodetector (ThorLabs, PDA55, rise time $50 \mathrm{ns)}$ giving sequential PA emissions recorded at a rate of $10 \mathrm{~Hz}$, before, during and after the HIFU exposure.

\section{Signal processing}

Conventional processing would involve extracting the amplitude of the PA signal from each radio-frequency segment measured using the PR transducer. The variation in this amplitude would be expected to track the change in the local temperature. However, a high level of system- and cavitation-related noise (Sec. III. A) hindered this implementation. Therefore, an alternate scheme was developed to extract the temperature information based on singular-value-decomposition (SVD). The hypothesis for using SVD is that distinct eigenvectors can track temperature information, cavitation noise, and system noise.

The PR signals corresponding to each experimental run were lumped into a matrix and SVD was performed. The SVD decomposition of this matrix echoes onto this SVD-derived base of eigenvectors is statistically optimal in the 


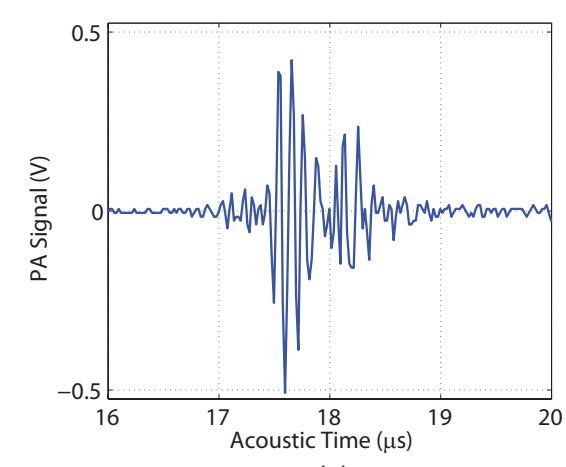

(a)

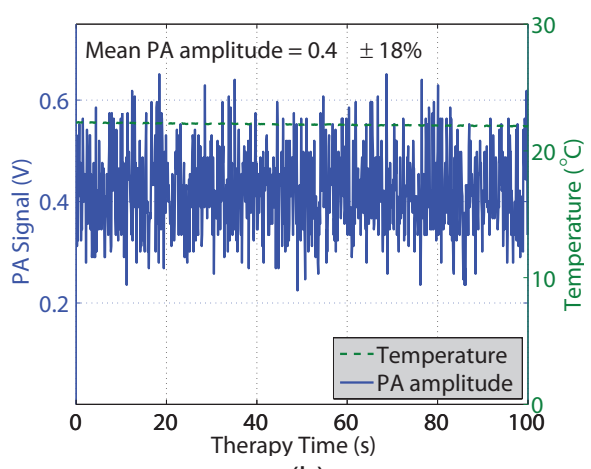

(b)

FIGURE 2. (a) A representative PA waveform received by the PR transducer. The amplitude of this signal tracks changes in local temperature; (b) The PA amplitude (from 1000 segments) and temperature measured over a duration of $100 \mathrm{~s}$ without HIFU. Measurements exhibit a standard deviation of $18 \%$ indicating large variability in shot-to-shot PA amplitude.

sense that the greatest variance by any projection of the data is represented by the first eigenvector, the second greatest variance by the second eigenvector, and so on [9]. These variances are equal to the square of the diagonal values of the eigenvalue matrix produced by SVD. The eigenvectors associated with system noise were identified as those beyond $90 \%$ of the total variance. Among the remaining eigenvectors (usually 7), the eigenvector whose decomposition coefficients (i.e., the coefficients of the orthogonal projection of the PR signals onto this eigenvector) contained the most energy after low-pass filtering was selected as the one containing temperature information (without broadband cavitation noise). The low-pass filter employed in this case was a 5-pt moving average filter (-6 dB cutoff at $1 \mathrm{~Hz})$. Then, a temperature profile was obtained by linear fitting the decomposition coefficients of the selected eigenvector to the thermocouple data. Finally, the remaining SVD eigenvectors not associated with system noise or temperature (usually 6) were used to quantify inertial cavitation. Specifically, the PR signals were projected onto the vector space formed by these eigenvectors, then the energy of the projections were calculated. This energy value was associated with cavitation.

\section{RESULTS}

Local temperature measurements using the thermocouple and the measurements made using the PR transducer were obtained before, during, and after each HIFU exposure (a total of 1000 waveforms). For all the results presented below, "acoustic time" refers to the propagation time for the acoustic (PA) wave and "therapy time" refers to the duration of an experimental run.

\section{Noise characterization}

System noise was characterized by monitoring PA waveforms and temperature over a duration of $100 \mathrm{~s}$ (1000 PA segments) without a HIFU-induced temperature elevation. A representative PA waveform is shown in Fig. 2(a). The amplitude of this signal depends on the local temperature and can be used to track changes in local temperature. Figure 2(b) shows the amplitude of the PA signals and illustrates the system noise. Measurements indicated that at a constant temperature of $23^{\circ} \mathrm{C}$, the PA signal amplitude exhibited a standard deviation of $18 \%$ around the mean, indicating large variability in shot-to-shot PA amplitude.

\section{PA-based temperature}

Figure 3 shows a representative measurement illustrating the change in PA amplitude due to a HIFU-induced temperature change and the corresponding temperature change measured by a thermocouple. The PA amplitude exhibits 


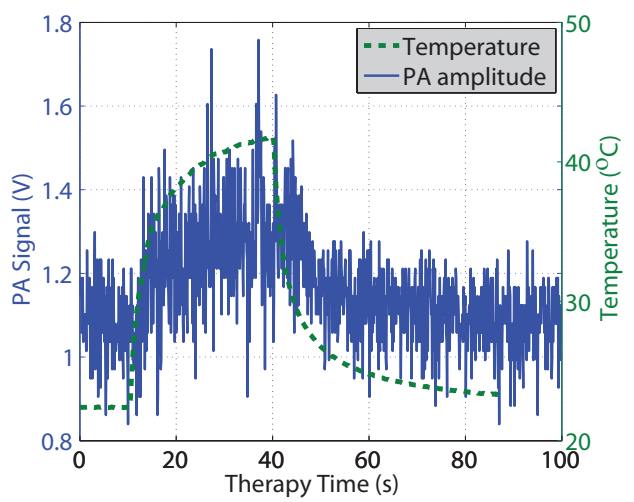

FIGURE 3. A representative measurement illustrating the change in PA amplitude due to HIFU-induced temperature change and the corresponding thermocouple measurement.

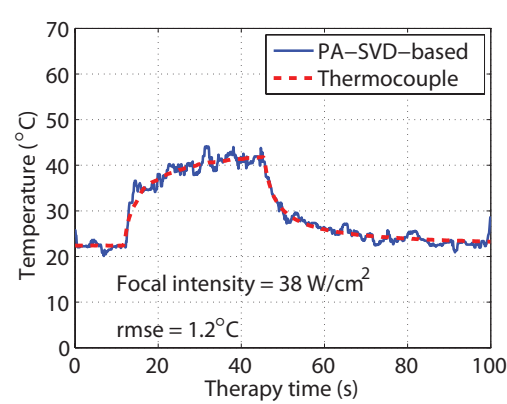

(a)

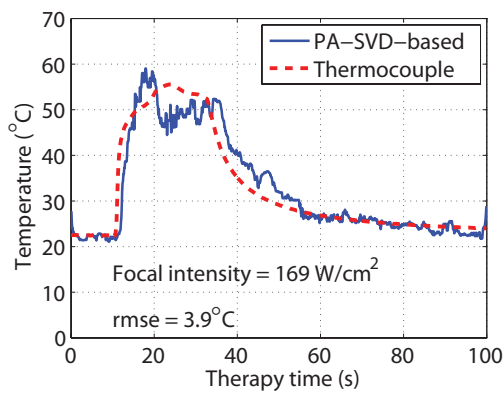

(c)

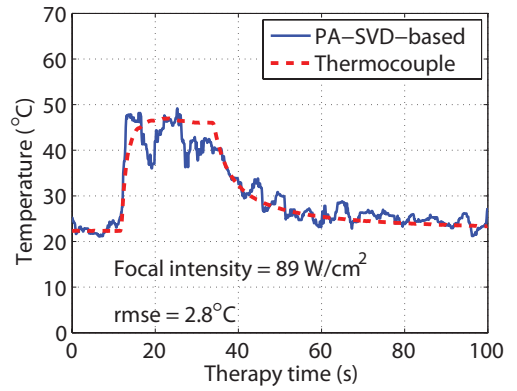

(b)

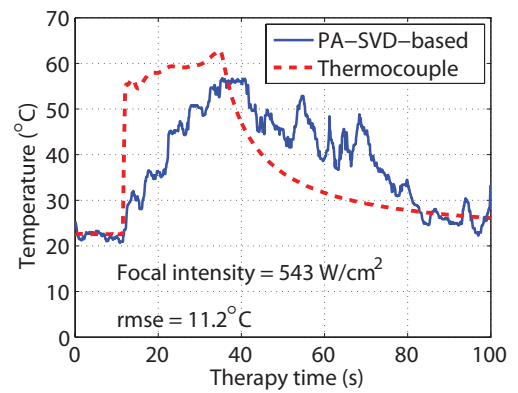

(d)

FIGURE 4. PA-SVD-based temperature profiles and corresponding thermocouple measurements for HIFU exposures at (a) $38 \mathrm{~W} / \mathrm{cm}^{2}$, (b) $89 \mathrm{~W} / \mathrm{cm}^{2}$, (c) $169 \mathrm{~W} / \mathrm{cm}^{2}$, and (d) $543 \mathrm{~W} / \mathrm{cm}^{2}$. PA-temperature curves exhibit close agreement with the thermocouple measurement for focal intensities less than $200 \mathrm{~W} / \mathrm{cm}^{2}$.

qualitative agreement with the thermocouple measurements and tracks the heating and the cooling phases associated with the HIFU exposure. The post-processed, PA-SVD-based temperature profile derived from this measurement and the corresponding thermocouple measurement are shown in Fig. 4(a). The SVD-based scheme effectively removes system and cavitation noise, and the remaining component from the measurement tracks the change in temperature. Processed temperature profiles at focal intensities of $89 \mathrm{~W} / \mathrm{cm}^{2}, 169 \mathrm{~W} / \mathrm{cm}^{2}$, and $543 \mathrm{~W} / \mathrm{cm}^{2}$ are shown in Fig. 4(b-d).

The SVD-deduced temperature profiles based on the PA amplitude correlated well with changes in temperature measured by the thermocouple, particularly for the exposure at intensities lower than $200 \mathrm{~W} / \mathrm{cm}^{2}$. The root-meansquared-error (rmse) deduced from the PA-temperature linear fit for these three HIFU intensities was less than $5^{\circ} \mathrm{C}$. In the case of the HIFU exposure at $543 \mathrm{~W} / \mathrm{cm}^{2}$, the PA-SVD-based temperature profile did provide indication of the gross temperature changes associated with HIFU exposures, but failed to track the temperature measured by the 


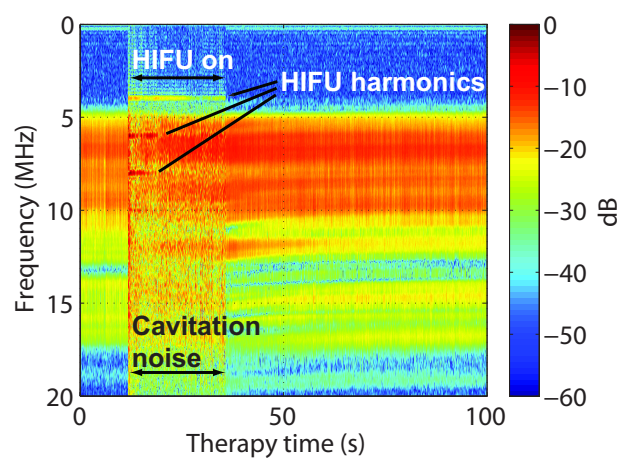

FIGURE 5. Spectral content of the PA data acquired during the $543 \mathrm{~W} / \mathrm{cm}^{2} \mathrm{HIFU}$ exposure. Presence of broadband noise during sonication indicated inertial cavitation.

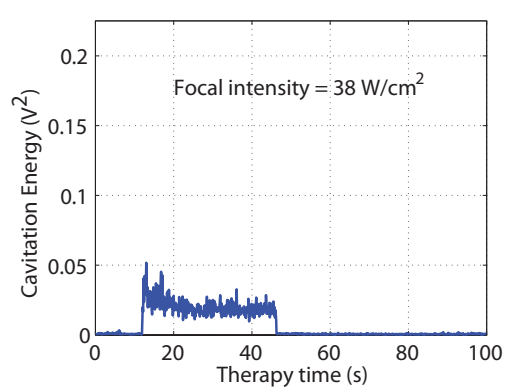

(a)

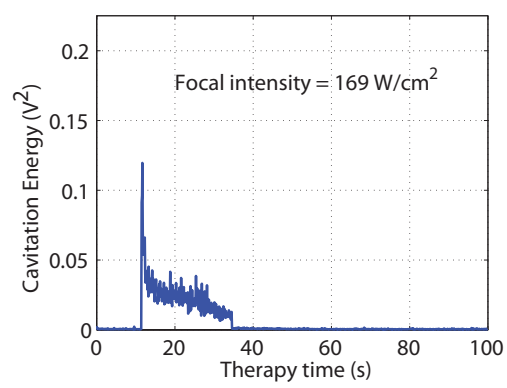

(c)

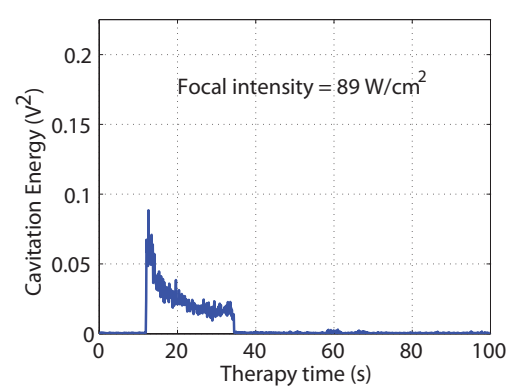

(b)

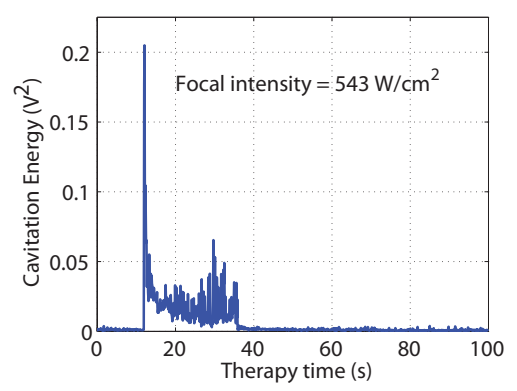

(d)

FIGURE 6. SVD-deduced inertial cavitation energy in the measured PR signals for HIFU exposures at (a) $38 \mathrm{~W} / \mathrm{cm}^{2}$, (b) $89 \mathrm{~W} / \mathrm{cm}^{2}$, (c) $169 \mathrm{~W} / \mathrm{cm}^{2}$, and (d) $543 \mathrm{~W} / \mathrm{cm}^{2}$. Inertial cavitation onset is synchronous with HIFU exposures and cavitation energy increases with increase in HIFU intensity.

embedded thermocouple $\left(\mathrm{rmse}=11.2^{\circ} \mathrm{C}\right)$.

\section{Cavitation results}

The spectral content of the PA data acquired during the $543 \mathrm{~W} / \mathrm{cm}^{2}$ HIFU exposure is shown in Fig. 5. For HIFU exposures at all intensities, the PR receiver detected broadband ultrasonic energy characteristic of inertial cavitation, which was present during the duration of HIFU exposure. The harmonics associated with the HIFU sonication centered at $2 \mathrm{MHz}$ also were apparent in the raw spectral data at focal intensities of $89 \mathrm{~W} / \mathrm{cm}^{2}$ and greater.

SVD-based processing yielded the inertial cavitation component of the signal received by the PR transducer. HIFU exposures at all four intensities resulted in inertial cavitation as shown in Fig. 6. Cavitation energy increased with 
focal intensity. A pattern of a sharp onset followed by decline in energy (typically associated with cavitation-induced "shielding") was observed. This behavior was more pronounced at higher intensities (Fig. 6(b-d)). It commonly is attributed to bubbles created by inertial cavitation, which hinder the trailing HIFU wave from reaching the focus.

\section{DISCUSSION AND CONCLUSION}

The SVD-based signal processing scheme was able to extract the temperature profile effectively despite the presence of noise in the PA measurements. Results indicated that for HIFU exposures at focal intensities lower than $200 \mathrm{~W} / \mathrm{cm}^{2}$, the dependence of the PA amplitude on temperature was described adequately using a linear fit, which is consistent with previous findings [7]. The PA-based sensor potentially can provide a congruent platform for monitoring in situ temperature and inertial cavitation during HIFU therapy as long as cavitation bubbles do not prevent the PA wave from reaching the PR transducer.

Interleaving HIFU exposure and PA measurements possibly could further alleviate cavitation and harmonics-based noise and facilitate noninvasive options for therapeutic hyperthermia with real-time temperature monitoring. The proposed scheme could be further augmented by use of targeted gold nanorods [10] allowed to accumulate in the tumors through bioconjugation and extravasation, which would enhance the signal-to-noise ratio of the PA wave emanating from the tumor.

\section{ACKNOWLEDGMENTS}

This research was supported by the Riverside Research Institute Biomedical Engineering Research Fund.

\section{REFERENCES}

1. G. T. Haar and C. C. Coussios. High intensity focused ultrasound: past, present and futures. International Journal of Hyperthermia, 23(2):85-87, 2007.

2. G. T. Clement. Perspectives in clinical uses of high-intensity focused ultrasound. Ultrasonics, 42:1087-1093, 2004.

3. T. A. Leslie and J. E. Kennedy. High-intensity focused ultrasound principles, current uses, and potential for the future. Ultrasound Quarterly, 22(4):263-272, 2006.

4. K. Hynynen, O. Pomeroy, and D. N. Smith. MR-imaging-guided focused ultrasound surgery of fibroadenomas in the breast: a feasibility study. Radiology, 219:176-185, 2001.

5. R. Mass-Moreno, C. A. Damianou, N. T. Sanghvi. Noninvasive temperature estimation in tissue via ultrasound echo-shifts. Part II. In vitro study Journal of the Acoustical Society of America, 100:2522-2530, 1994.

6. A. Anand and P. Kaczkowski. Noninvasive measurement of local thermal diffusivity using backscattered ultrasound and focused ultrasound heating. Ultrasound in Medicine \& Biology, 34(9):1449-1464, 2008.

7. R. Esenaliev, A. Oraevsky, K. Larin, I. Larina, and Motamedi. Real-time optoacoustic monitoring of temperature in tissues. Photochemical, Photothermal, and Photomechanical, SPIE, 3601:268-275, 1999.

8. M. Xu and L. Wang. Photoacoustic imaging in biomedicine. Review of Scientific Instruments, 77(4): 041101-22, 2006.

9. J. E. Gentle. Numerical linear algebra for applications in statistics. Statistics and computing, Springer, New York, 1998.

10. R. Rayavarapu, W. Petersen, C. Ungureanu, J. Post, T. Leeuwen, and S. Manohar. Synthesis and bioconjugation of gold nanoparticles as potential molecular probes for light-based imaging techniques. International Journal of Biomedical Imaging, 298171-10, 2007. 